\title{
Observation of Candida albicans treated with clioquinol under fluorescence and scanning electron microscope
}

\author{
Zimeng You ${ }^{1}$, Xin Ran ${ }^{1}$, Chaoliang Zhang ${ }^{2}$, Yuping Ran ${ }^{*}$
}

\section{${ }^{1}$ Department of Dermatovenereology, West China Hospital, Sichuan University, ${ }^{2}$ State Key Laboratory of Oral Diseases, West China Stomatology Hospital, Sichuan University, Chengdu 610041, China "Corresponding: ranyuping@vip.sina.com}

Introduction: Clioquinol was firstly produced in 1934 as antiseptic. The target of antifungal function was unknown though apoptosis, autophagy, cell cycle arrest may be associated with the antineurodegenerative diseases and anti-tumor function ${ }^{1,2}$. We confirmed the antifungal function of clioquinol was strong and broad in former experiment of our team.

Objective and Methods: We tried to find the clue of target through morphologic change after Candida albicans treated with clioquinol at 0.5 minimum inhibitory concentration (MIC) for 7 days. We used agar dilution method to measure MIC of active pharmaceutical ingredient (API). Slide culture was made on potato dextrose agar (PDA) with API added at concentration of $0.5 \mathrm{MIC}$. Control groups included blank and solvent control. The observation under fluorescence (FM) and scanning electron microscope (SEM) was conducted after 7 days.

Results: MIC of all drugs tested for C. albicans was listed in Fig. 1 Fluorescence intensity was very strong and multiple hyphae formed after 7-day culture in blank and solvent control group both in ATCC10231 and clinical strain. When observed under SEM, the surface of spores was smooth and no depression and fissures were seen in control groups. As for ATCC10231, fluorescence intensity weakened and hyphae formation decreased in clioquinol group (4 $\mu \mathrm{g} / \mathrm{ml})$. Surface depressed in parts of spores with no fissures under SEM (Fig. 2). When the same method was used in $C$. albicans clinical strain, no obvious changes in clioquinol group at $4 \mu \mathrm{g} / \mathrm{ml}$ were seen under FM compared with control groups.
Though surface of few spores depressed under SEM was observed. Obvious decrease of fluorescence intensity and hyphae formation was found in clioquinol group at $8 \mu \mathrm{g} / \mathrm{ml}$. As for observation under SEM, most spores depressed and distinct fissures seen in parts of spores (Fig. 3).

Discussion: When $C$. albicans was treated with clioquinol at 0.5 MIC, obvious changes in morphology were observed under FM and SEM. The target of antifungal function of clioquinol may be related to synthesis of cell wall. We still need more data based on different species to confirm the finding in $C$. albicans. Since the increase of drug-resistant strains, new effective antifungal drugs development is significant and meaningful. Clioquinol can be a promising drug in treating infective diseases though more data based on mechanism and clinical work are needed.

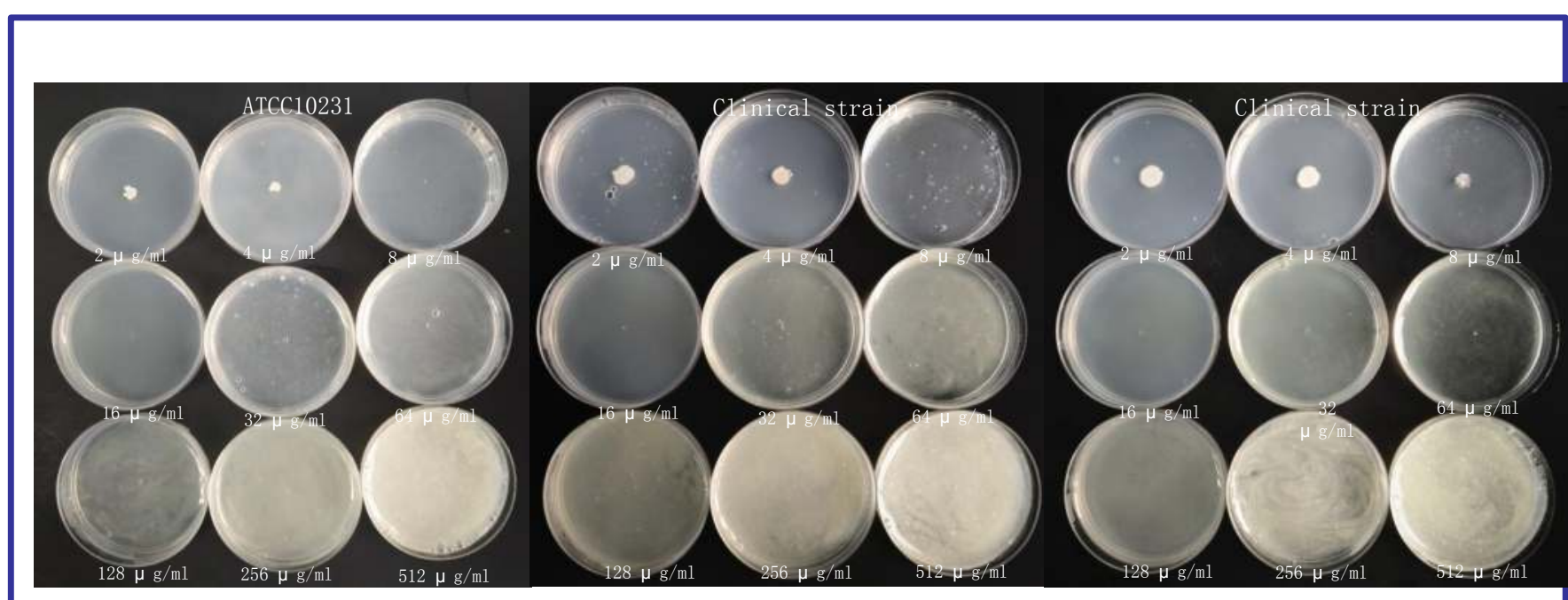

Fig.1. Minimum inhibitory concentration (MIC) of clioquinol for $C$ albicans.

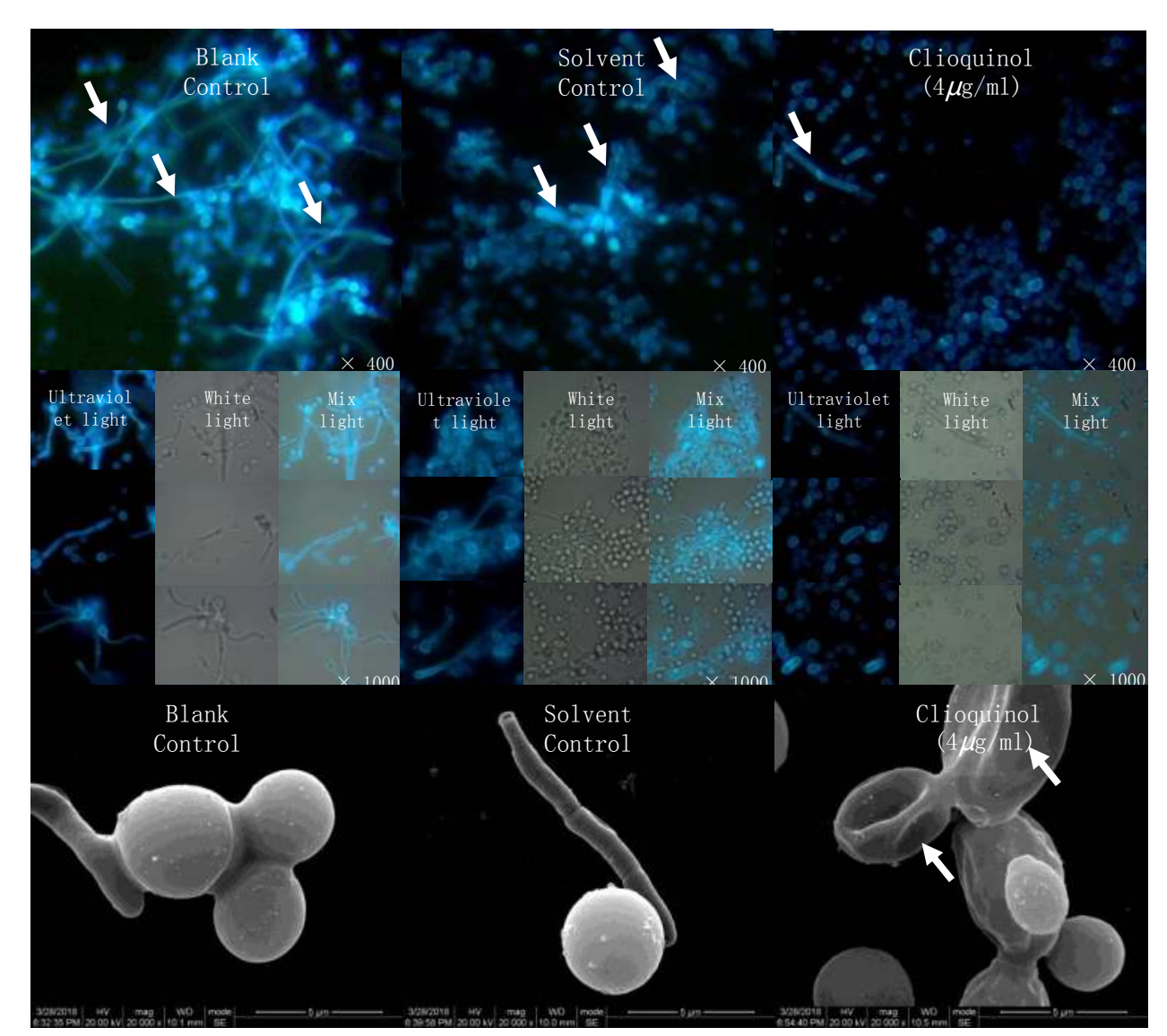

Fig. 2. Morphology change of C. albicans ATCC10231.

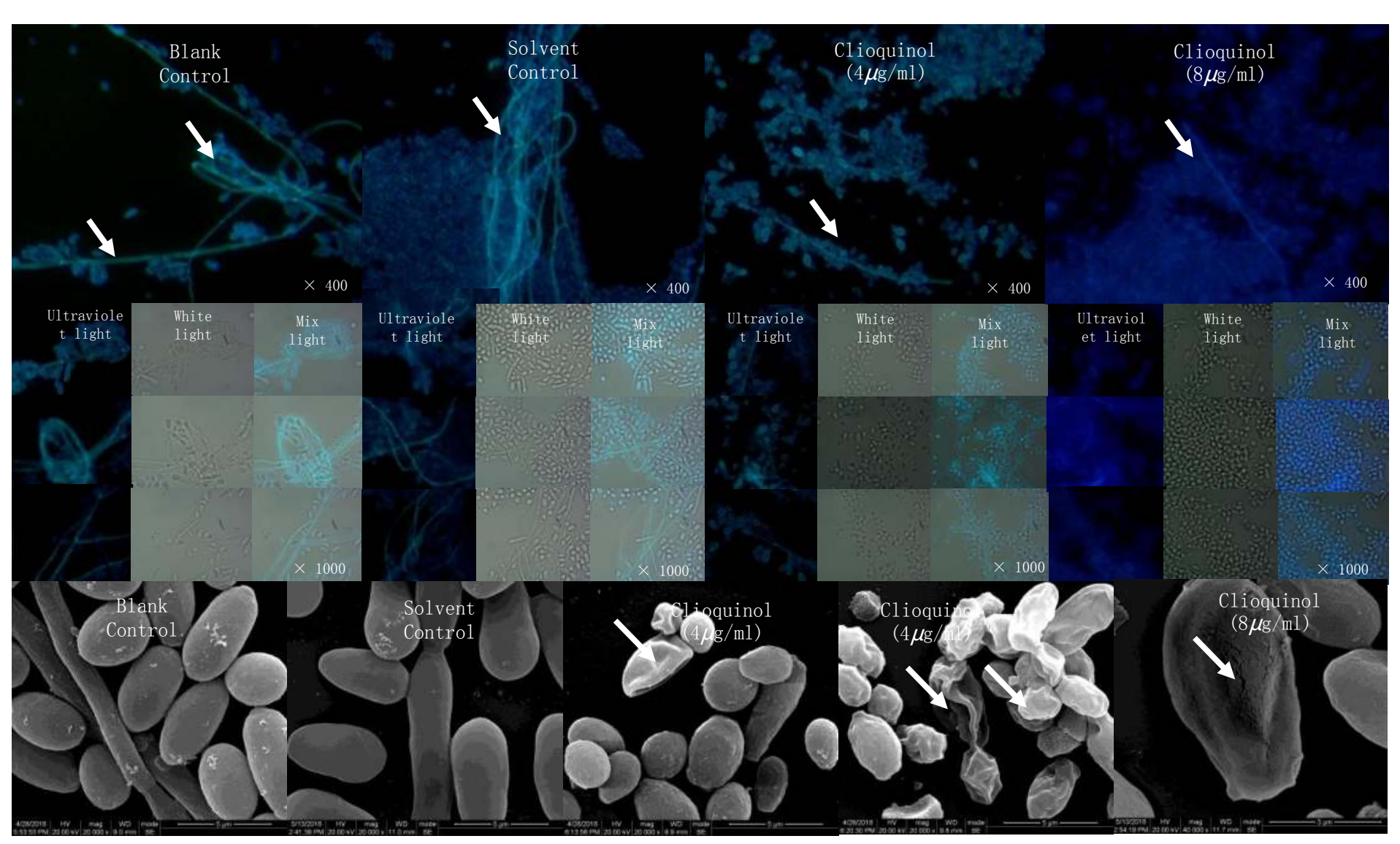

Fig. 3. Morphology change of $C$. albicans clinical strain.

\section{$\underline{\text { References }}$}

1. Bareggi SR, Cornelli U. Clioquinol: review of its mechanisms of action and clinical uses in neurodegenerative disorders. CNS Neurosci Ther, 2012;18(1):41-6.

2. He ML, Luo M, Liu QY, et al. Combination treatment with fasudil and clioquinol produces synergistic anti-tumor effects in U87 glioblastoma cells by activating apoptosis and autophagy. J Neurooncol. 2016;127(2):261-70. 